\title{
The Arabic origins of "Love and Sexual Terms" in English and European languages: A lexical root theory approach
}

\author{
Zaidan Ali Jassem \\ Department of English Language and Translation, Qassim University, P.O.Box 6611, Buraidah, KSA
}

Email address:

zajassems@gmail.com

To cite this article:

Zaidan Ali Jassem. The Arabic Origins of "Love and Sexual Terms" in English and European Languages: A Lexical Root Theory Approach. International Journal of Language and Linguistics. Vol. 1, No. 4, 2013, pp. 97-110. doi: 10.11648/j.ij11.20130104.13

\begin{abstract}
This paper investigates the Arabic cognates or origins of love and sexual words in English, German, French, Latin, and Greek from a lexical root theory perspective. The data consists of 239 terms like love, hope, abhor, hate, cupid, woo, whore, slut, fuck, erotic, intercourse, sex, copulate, impregnate, fornicate, marry, wed, seduce, beautiful, and so on. The results demonstrate that all such words have true Arabic cognates, having the same or similar forms and meanings. Their different forms, however, are all shown to be the result of natural and plausible causes and courses of linguistic change in such languages. For example, English, French, Greek and Latin erotic (Eros) comes from Arabic 'arr 'intercourse, making love'; English, French, and Latin abhor obtains from Arabic kariha/'akrah, kurh (n) 'hate' via /k \& h/-merger; English and German love/lieben derives from Arabic labba ('alabba) 'to love, live/stay', turning / $\mathrm{b} /$ into /v/; English hope (hobby) and German hoffen is from Arabic $2 u b b$ 'love, hope', turning /2/ into /h/ and /b/ into /f/ in the latter. Consequently, the results indicate, contrary to Comparative Method claims, that Arabic, English and all (Indo-)European languages belong to the same language, let alone the same family. They, therefore, prove the adequacy of the lexical root theory according to which Arabic, English, German, French, Latin, and Greek are dialects of the same language with the first being the origin because of its phonetic complexity, huge lexical variety and multiplicity with over 100 'sex' terms.
\end{abstract}

Keywords: Love \& Sexual Words, Arabic, English, German, Latin, Greek, Historical Linguistics, Lexical Root Theory, French

\section{Introduction}

The lexical root theory (Jassem 2012a-f, 2013a-p) derives its name from using lexical roots in tracing genetic relationships between words in world languages. It first arose as a rejection of the classification of the Comparative Method in historical linguistics that Arabic belongs to a different language family from English, German, French, and all (Indo-) European languages in general (Bergs and Brinton 2012; Algeo 2010; Crystal 2010: 302; Campbell 2006: 190-191; Yule 2006; Crowley 1997: 22-25, 110-111; Pyles and Algeo 1993: 61-94). On the contrary, it clearly demonstrated the inextricably close, genetic relationship between Arabic and such languages phonetically, morphologically, grammatically, and semantically or lexically (Jassem 2012a-f, 2013a-p).

Twenty two studies have already been undertaken on all language levels. Lexically, fourteen studies successfully traced the Arabic origins of English, German, French, Latin, Greek and Sanskrit words in key semantic fields- namely, numeral words (Jassem 2012a), common religious terms (Jassem 2012b), water and sea terms (Jassem 2013d), air and fire terms (Jassem 2013e), celestial and terrestrial terms (Jassem 2013f), animal terms (Jassem (2013g), body part terms (Jassem 2013h), speech and writing terms (Jassem 2013i), time words (Jassem 2013j), family words (Jassem 2013k), cutting and breaking words (Jassem 2013m), movement and action words (Jassem 2013n), perceptual and sensual words (Jassem 2013o), and cognitive and mental words (Jassem 2013p). Morphologically, three studies established the Arabic origins of English, German, French, Latin, and Greek inflectional 'plural and gender' markers (Jassem 2012f), derivational morphemes (Jassem 2013a), and negative particles (Jassem 2013b). Grammatically, four papers described the Arabic origins of English, German, French, Latin, Greek, and Sanskrit personal pronouns (Jassem 2012c, 20131), determiners (Jassem 2012d), and verb 'to be' forms (Jassem 2012e). Phonetically, Jassem (2013c) outlined the English, German, French, Latin, and Greek cognates of Arabic back consonants: viz., the glottals, pharyngeals, uvulars, and velars. Besides, the phonetic 
analysis recurs in all the papers, of course. In all such studies, Arabic and English words, for example, were true cognates with similar or identical forms and meanings.

The rest of this paper consists of four sections: (i) research methods, (ii) results, (iii) discussion, and (iv) conclusion.

\section{Research Methods}

\subsection{The Data}

The data consists of 239 love and sexual words such as love, hope, abhor, hate, cupid, woo, whore, slut, fuck, erotic, intercourse, sex, copulate, impregnate, fornicate, marry, wed seduce, beautiful, and so on. Their selection has been based on the author's knowledge of their frequency and use and English dictionaries and thesauri. For easy reference, they will be arranged alphabetically together with brief linguistic comments in (3.) below.

Regarding etymological data for English and European languages, all references are for Harper (2012); for Arabic, lexical or semantic data are for Altha3aalibi (2011: 206-14), Ibn Seedah (1996: 4/13-20, 59-63; 5/110-15), and Ibn Manzoor (2013) in the main.

In transcribing the data, normal spelling is used for practical purposes; nevertheless, certain symbols were used for unique Arabic sounds, including / 2 \& 3 / for the voiceless and voiced pharyngeal fricatives respectively, $/ \mathrm{kh} \& \mathrm{gh} /$ for the voiceless and voiced velar fricatives each, capital letters for the emphatic counterparts of plain consonants $/ \mathrm{t}, \mathrm{d}, \mathrm{dh}, \&$ S/, and /'/ for the glottal stop (Jassem 2013c).

The above love and sexual words can constitute fully natural texts on their own in today's English, e.g.,

Valentino: I hope that you that love me, Aphrodite.

Aphrodite: I don't hate you, Valentino.

Casanova: She abhors, detests, and despises you.

Amy: That's disgustingly seductive.

Erasmus: Wish you kissed me erotically once, Aphrodite.

Amy: That's rude and lewd, lascivious and voluptuous, Erasmus.

Casanova: That's lecherous.

Every word in the above fully natural English text has a true Arabic cognate as will be shown in the analysis below.

\subsection{Data Analysis}

\subsubsection{Theoretical Framework: The Lexical Root Theory}

The data will be analyzed by utilizing the lexical root theory as a theoretical framework (Jassem 2012a-f, 2013a-p). It is so called because of employing the lexical (consonantal) root in examining genetic relationships between words such as the derivation of observation from serve (or simply srv). The major reason stems from the fact that the consonantal root carries and determines the basic meaning of the word irrespective of its affixation such as observation. Historically speaking, classical and modern Arabic dictionaries (e.g., Ibn Manzoor 1974, 2013) used consonantal roots in listing lexical entries, a practice first founded by Alkhaleel, an $8^{\text {th }}$ century linguist, lexicographer, musician, and mathematician (Jassem 2012e).

The lexical root theory has a simple structure, which comprises a theoretical principle or hypothesis and five practical procedures of analysis. The principle states that:

Arabic and English as well as the so-called Indo-European languages are not only genetically related but also are directly descended from one language, which may be Arabic in the end. In fact, it claims in its strongest version that they are all dialects of the same language, whose differences are due to natural and plausible causes and courses of linguistic change.

To empirically prove that, five applied procedures are used in data collection and analysis: namely, (i) methodological, (ii) lexicological, (iii) linguistic, (iv) relational, and (v) comparative/historical. As all have been reasonably described in the above studies (Jassem 2012a-f, 2013a-n), a brief summary will suffice here.

Firstly, the methodological procedure concerns data collection, selection, and statistical analysis. Apart from loan words, all language words, affixes, and phonemes are amenable to investigation, and not only the core vocabulary as is the common practice in the field (Crystal 2010; Pyles and Algeo 1993: 76-77; Crowley 1997: 88-90, 175-178). However, data selection is practically inevitable since no single study can accomplish that at one time, no matter how ambitious it might be. The most appropriate way for approaching that goal would be to use semantic fields such as the present and the above topics. Cumulative evidence from such findings will aid in formulating rules and laws of language change at a later stage (cf. Jassem 2012f, 2013a-f). The statistical analysis employs the percentage formula (see 2.2 below).

Secondly, the lexicological procedure is the initial step in the analysis. Words are analyzed by (i) deleting affixes (e.g., explained $\rightarrow$ plain), (ii) using primarily consonantal roots (e.g., plain $\rightarrow$ pln), and (iii) search for correspondence in meaning on the basis of word etymologies and origins as a guide (e.g., Harper 2012), to be used with discretion, though. The final outcome yields Arabic baien, baan (v) 'clear, plain' via /1/-insertion or split from $/ \mathrm{n} /$ (Jassem 2013i).

Thirdly, the linguistic procedure handles the analysis of the phonetic, morphological, grammatical and semantic structures and differences between words. The phonetic analysis examines sound changes within and across categories. In particular, consonants may change their place and manner of articulation as well as voicing. At the level of place, bilabial consonants $\leftrightarrow$ labio-dental $\leftrightarrow$ dental $\leftrightarrow$ alveolar $\leftrightarrow$ palatal $\leftrightarrow$ velar $\leftrightarrow$ uvular $\leftrightarrow$ pharyngeal $\leftrightarrow$ glottal (where $\leftrightarrow$ signals change in both directions); at the level of manner, stops $\leftrightarrow$ fricatives $\leftrightarrow$ affricates $\leftrightarrow$ nasals $\leftrightarrow$ laterals $\leftrightarrow$ approximants; and at the level of voice, voiced consonants $\leftrightarrow$ voiceless.

In similar fashion, vowels change as well. Although the number of vowels differ greatly within and between English (Roach 2008; Celce-Mercia et al 2010) and Arabic (Jassem 2012g, 1987, 1993), all can be reduced to three basic long 
vowels /a: (aa), i: (ee), \& u: (oo)/ (and their short versions besides the two diphthongs /ai (ay)/ and /au (aw)/ which are a kind of /i:/ and /u:/ respectively). They may change according to modifications in (i) tongue part (e.g., front $\leftrightarrow$ centre $\leftrightarrow$ back), (ii) tongue height (e.g., high $\leftrightarrow$ mid $\leftrightarrow$ low), (iii) length (e.g., long $\leftrightarrow$ short), and (iv) lip shape (e.g., round $\leftrightarrow$ unround). In fact, the vowels can be, more or less, treated like consonants where $/ \mathrm{i}$ :/ is a kind of $/ \mathrm{j}(\mathrm{y}) /, / \mathrm{u}: / \mathrm{a}$ kind of $/ \mathrm{w} /$, and /a:/ a kind of $/ \mathrm{h} /$ or vice versa. Their functions are mainly phonetic such as linking consonants to each other in speech and grammatical such as indicating tense, word class, and number (e.g., sing, sang, sung, song; man/men). Thus their semantic weight is little, if not at all. For these reasons, vowels are marginal in significance which may be totally ignored in the analysis because the limited nature of the changes do not affect the final semantic result at all.

Sound changes result in natural and plausible processes like assimilation, dissimilation, deletion, merger, insertion, split, syllable loss, re-syllabification, consonant cluster reduction or creation and so on. In addition, sound change may operate in a multi-directional, cyclic, and lexically-diffuse or irregular manner (for detail, see Jassem 2012a-f, 2013c).

Regarding the morphological and grammatical analyses, some overlap obtains. The former examines the inflectional and derivational aspects of words in general (Jassem 2012f, 2013a-b); the latter handles grammatical classes, categories, and functions like determiners, pronouns, nouns, verbs, and case (Jassem 2012c-e). Since their influence on the basic meaning of the lexical root is marginal, they may also be ignored altogether.

As regards the semantic analysis, it examines meaning relationships between words, including lexical stability, multiplicity, convergence, divergence, shift, split, change, and variability. Stability means that word meanings have remained constant over time. Multiplicity denotes that words might have two or more meanings. Convergence means two or more formally and semantically similar Arabic words might have yielded the same cognate in English. Divergence signals that words became opposites or antonyms of one another. Shift indicates that words switched their sense within the same field. Lexical split means a word led to two different cognates. Change means a new meaning developed. Variability signals the presence of two or more variants for the same word (for detail, see Jassem 2012a-f).

Fourthly, the relational procedure accounts for the relationship between form and meaning from three angles: formal and semantic similarity (e.g., three, third, tertiary and Arabic thalath 'three' (Damascus Arabic talaat (Jassem 2012a)), formal similarity and semantic difference (e.g., ship and sheep (Jassem 2012b), and formal difference and semantic similarity (e.g., quarter, quadrant, cadre and Arabic qeeraaT '1/4' (Jassem 2012a)).

Finally, the comparative historical analysis compares every word in English in particular and German, French, Greek, and Latin in general with its Arabic counterpart phonetically, morphologically, and semantically on the basis of its history and development in English (e.g., Harper 2012; Pyles and Algeo 1993) and Arabic (e.g., Ibn Manzour 2013; Altha3aalibi 2011; Ibn Seedah 1996) besides the author's knowledge of both Arabic as a first language and English as an equal second language. Discretion should be exercised here due to uncertainties and inaccuracies, especially in Harper's work, though.

\subsubsection{Statistical Analysis}

The percentage formula is used for calculating the ratio of cognate words or shared vocabulary, which is obtained by dividing the number of cognates over the total number of investigated words multiplied by a 100. For example, suppose the total number of investigated words is 100 , of which 90 are true cognates. The percentage of cognates is calculated thus: $90 / 100=9 \times 100=90 \%$. Finally, the results are checked against Cowley's (1997: 173, 182) formula to determine whether such words belong to the same language or family (for a survey, see Jassem 2012a-b).

\section{Results}

The main focus of the results will be on the Arabic lexical (consonantal) roots of English, German, French, Latin, and Greek words. This entails that affixation (prefixes, suffixes, and infixes) are generally excluded to save on time, space, and effort here although all have true Arabic cognates (see Jassem 2012f, 2013a).

Abhor (abhorrence, abhorrent) via Latin abhorrere 'shudder, tremble at, be averse to' of (i) $a b$ 'off, away from' from Arabic $b i$ 'in, with' or $a a b$, iaab 'coming back' via lexical divergence and (ii) horrere from Arabic kariha, kurh (n) 'hate' where /k \& h/ merged into /h/ (Jassem 2013c).

Abominable (abomination) via Latin abominare of (i) $a b$ above and (ii) omen 'foreboding' from Arabic yameen/yumn 'right, good foreboding, safety', amina (v) 'be safe' via lexical shift.

Accept (acceptable) via Latin adcapere of (i) ad 'to' from Arabic 2atta 'to, until' via /2/-deletion and turning /t/ into /d/ and (ii) capere 'take' from Arabic jaab 'bring' via lexical shift and changing $/ \mathrm{j} /$ into $/ \mathrm{k} /$; or $2 a b b a d h$ 'like, accept' via $/ 2 \& \mathrm{dh} /$-mutation into $/ \mathrm{ks} \& \mathrm{t} /$.

Adolescent (adult) via Latin adolescere 'grow up, mature, ripen' from Arabic Tala3 'appear, grow up' where /T \& $3 /$ became /t \& s/ or 3adala 'to grow up, to be just', $3 a d l$ 'a just person; fat' via /3/-loss.

Adore (adoration, adorable) via Latin adorare 'to speak' of ad 'to' from Arabic 2atta 'to' via /2/-loss and turning /d/ into /t/ and orare 'speak' from Arabic war, warwar 'cry, speak' (Jassem 2013i); Dara3a, taDarra3a 'beseech, ask humbly, adore' in which /D \& 3/ turned into /d \& Ø/; or dalaal, dallal (v) 'to treat very kindly, to pamper' where $/ \mathrm{l} /$ became $/ \mathrm{r} /$.

Adult (adolescent) via Latin adultus, adolescere (v) 'grown up, mature, ripe' from Arabic Tala3, Taali3(at) 'appear, 
grow up' where $/ \mathrm{T} \& 3 /$ turned into $/ \mathrm{t} \& \varnothing /$; or 3 adala 'to grow up, to become fat, to be just', 3 adil(at)/3adl(at) (adj.) 'a just person; fat' via /3/-loss

Adultery (adulterer, adulteress) via French avoutrie and Latin adulterare 'to corrupt' from Arabic 3aaTil(at) 'faithless, lewd, useless', deleting /3/ and turning /T/ into $/ \mathrm{t} /$; 3aaDhal(at) 'of dogs, to mate' via $/ 3 /$-loss and changing $/ \mathrm{Dh} /$ to $/ \mathrm{d} /$.

Affable (affability) via Latin affare of $a b$ above + fare 'speak' from Arabic affa 'refuse, breathe'; $f a^{\prime} f a^{\prime}$ 'to mutter' via syllable merger; or haffa/faha 'love, desire', deleting $/ \mathrm{h} /$.

Affair from Arabic 'amr 'affair, order'; /m/ became /f/.

Affection (affectionate, affect) from Arabic wajd 'love-sorrow', turning /w, j, \& d/ into /f, k, \& t/; or faqd 'sorrow, love; loss' where /q \& d/ becme /k \& t/.

Agree (agreement) from Arabic aqarra 'agree'; /q/ became $/ \mathrm{g} /$.

Allure (allurement) from Arabic la'la' 'shine' where /1/ became /r/; raa'a 'show off' where /1/ split from /r/, warra 'to show' where /w/ became /1/, or aghra 'seduce' via /gh \& r/-merger into /r/ from which /1/ split.

Amicable (amicability; Amy) via Latin amare 'to love' from Arabic haama, hiaam (n) 'to love madly', deleting /h/; or raama 'to love' via reversal. See enemy.

Anal (anus) from Arabic 3aana(t) 'anus' via /3/-loss or hann 'vagina' via lexical shift and /h/-loss (Jassem 2013h).

Andrew (Andy, Andy-dandy) via Latin/Greek Andreas 'manly' from Arabic 3 antar(at) 'a powerful, daring man' via $/ 3 /$-loss and $/ \mathrm{t} /$-evolution into $/ \mathrm{d} /$.

Animosity via Latin animosita 'boldness, vehemence' from Arabic 2amaasat 'vehemence, enthusiasm' via /2/-loss and /n/-split from /m/; or khaSm, khuSoomat (n) 'enemy, animosity' via reordering, merging $/ \mathrm{kh} \& \mathrm{~S} /$ into /s/, and /n/-insertion.

Aphrodisiac (Aphrodite) via Greek aphrodisiakos 'inducing sexual desire', aphrodisios 'sexual pleasure', Aphrodite 'love and beauty goddess' from Arabic furhud/fulhud, furhood 'a beautiful, full-bodied young boy or adolescent; a soft and tender boy; baby lion', faraheed (pl.) 'baby sheep' via lexical shift; rafood 'a woman given as a present to man' via reordering; or fahr, ifhaar, iftihaar 'having sex with a woman at home while another hearing them' via $/ \mathrm{h} /$-loss and changing $/ \mathrm{t} / \mathrm{to}$ $/ \mathrm{d} /$.

Approve (approbation) via Latin approbare 'regard something as good' of ad 'to' above and probare 'try, find something to be as good; probus 'honest, genuine' from Arabic rabba, rabeeb (adj.) 'bring up, rear, polish' via reordering and turning $/ \mathrm{b} /$ into $/ \mathrm{v} /$; ra22aba 'approve, welcome' via reordering and replacing $/ 2 /$ by $/ \mathrm{v} /$.

Arouse (arousal) from Arabic athaara 'stir, arouse' via reordering and turning /th/ into $/ \mathrm{s} /$.

Arse from Arabic sharj 'arse' via reordering and merging /sh \& j/ into /s/ (Jassem 2013h).

Aspire (aspiration) via French aspire and Latin aspirare 'hope' from Arabic Saba, aSboo 'hope, aspire' or ishra'abba 'long for, hope' via reordering and turning /S (sh)/ into /s/.

Ass from Arabic ist 'ass' where /s \& t/ merged into /s/; 2ush 'ass' via $/ 2 \& \mathrm{sh} /$-merger into /s/ (Jassem 2013h); ja2sh 'donkey' via /j, 2, \& sh/-merger into /s/ (Jassem 2013g); or sees/soos 'a woman's vagina's cleavage; a donkey's back; donkey' via lexical shift.

Averse (aversion) from Arabic zawara/azwar 'looking aside' via reordering and turning $/ \mathrm{z} \& \mathrm{w} /$ into $/ \mathrm{s} \& \mathrm{v} /$; or a3raDa 'be averse to'; $/ 3 \& \mathrm{D} /$ turned into $/ \mathrm{v} \& \mathrm{~s} /$.

Bachelor from Arabic bikr 'virgin', turning / $/$ into /ch/ and inserting $/ 1 /$.

Barren from Arabic baayer, boor(aan) 'unmarried, barren, useless'.

Bastard via French batard, fils de bast 'son of the bed, pack-saddled son' from Arabic (i) bisaaT 'rug, bed', bassaaT(at) '(a female) rug maker', turning /q/ into /d/ (-art/ard 'pejorative ending' from Arabic -at/-ant 'fem./mas. suffix' via /r/-insertion (Jassem 2013c); (ii) filu 'baby horse' via lexical shift (Jassem 2013g); (iii) $d h i$ 'of' where /dh/ became /d/ (Jassem 2012c).

Beauty (beautiful, beautify, beau) via French beau 'beautiful' from Arabic bahi(at), baahi(at) 'beautiful' via $/ \mathrm{h} /$-elision.

Bed (bedding, embed) from Arabic mahd 'bed' where /b \& h/ merged into $/ \mathrm{m} /$ or baTa2, inbaTa 2 'lie down' where $/ \mathrm{T}$ \& $2 /$ became $/ \mathrm{d} \& \emptyset /$.

Beseech (seek) from Arabic shawq 'longing', 3ishq 'love', or shabaq 'sex desire' via/3/-loss, turning /sh \& q/ into /s $\& \mathrm{ch} /$, and reordering.

Betroth (betrothal) from Arabic tarDiat '(bringing into) agreement, appeasement' via lexical shift and turning /D/ into /th/ or rafath 'intercourse, allusion to marriage' via reordering and turning /f/ into /t/.

Bitch from Arabic qa2ba(t) 'prostitute' via reordering, /2/-loss, and changing /q/ to /ch/ or kalb(at) 'a bitch, $\operatorname{dog}^{\prime}$ via reordering and turning $/ \mathrm{k} \& \mathrm{l} /$ into $/ \mathrm{ch} \& \mathrm{t} /$ (Jassem 2013g).

Boyfriend (friend) from Arabic (i) Sabi 'boy' where /S/ merged into /b/ and (ii) rafeeq, rufqaan ( $\mathrm{pl}$ ) 'friend', turning /q/ into /d/ (Jassem 2013k).

Bride (bridegroom) from Arabic rubaD, rabD 'bride, wife' via reordering and replacing /D/ by /d/ (Jassem 2013k).

Bridegroom from Arabic ghulaam 'a young bachelor', ightalam (v) 'to mature sexually'; /gh \& 1/ turned /g \& $\mathrm{r} /$.

Buggery (bugger) via French Bougre and Latin Bulgarus 'heretic, sodomite, Bulgarian' from Arabic baghi 'whore; unjust', baghaa' (n) 'prostitution' where /gh/ split into /g \& r/; baak/baaq 'of dogs, to mate', replacing /k/ by /g/ and inserting /r/; baaqir 'seditious; dissecting; cowboy', turning /q/ into /g/; or bajar 'evil' where /j/ became $/ \mathrm{g} /$.

Caress from Arabic qaraS 'sting'; /q \& S/ became /k \& s/.

Casanova 'a man of carnal desires' from Arabic 2asan, 2assoon 'a beautiful, good-looking man' where $/ 2 \& \mathrm{w}$ 
(oo)/ evolved into $/ \mathrm{k} \& \mathrm{v} /$.

Celibate (celibacy) from Arabic sallab(at) or shabal, ashabal(at) 'of a widowed woman, not to remarry for her kids' sake' via reordering and changing /sh/ to /s/ (Jassem 2013k).

Chaste (chastity, incest) via Latin castus 'pure; cut-off, separated' from Arabic qazaazat 'shyness, modesty', qazz(at) 'a nice, modest person, who shuns blemishes' via /q \& z/-mutation into /ch \& s/. (Cf. qashaT/kashaT 'to strip, separate' where /q \& sh/ changed to /s/.) See incest.

Chic (chi-chi) via French chic 'style, artistic skill' and German Schick 'tact, skill', schicken (v) 'arrange' from Arabic shakl 'form, style' via /1/-loss; kaiyes 'nice' or shaiyiq 'loveable' via /k \& s (q)/-mutation into /ch (s)/. See Jassem (2013h, k).

Cock (coquette) from Arabic qooq 'a long-necked water bird; a woman's vagina's cleavage; a tall, foolish man; the sound of chicken' via lexical shift and turning /q/ into /k/; or SooS(at) 'baby chicken', changing /S/ into /k/. See ass.

Coerce (coercion) from Arabic qasara 'coerce, force' via reordering and turning $/ \mathrm{q} /$ into $/ \mathrm{s} /$.

Co-habit (cohabitation, habitat, habitation, inhabitant, habit) as a compound of Latin co/com 'together' from Arabic (i) sawa 'together' where /s/ became /k/ and (ii) bait 'home, house', baata (v) 'live' or 'adab 'manners, politeness' via reordering and turning /' \& d/ into /h \& t/.

Concubine via Latin concubare 'lie together/with' as a combination of (i) con (com) 'all, together, with' from Arabic jamee3 'all, together' where /j \& 3/ became /k \& $\varnothing /$ or $m a 3 a$ 'with' via reversal and turning /3/ into $/ \mathrm{k} /$ and (ii) cubare 'lie with' from Arabic kaba/inkabba 'sleep, lie on one's belly or face down'; Saa2ib, Su2baan (pl.) 'companion' via /S \& 2/-merger into /k/; $2 i b b$ (aan) 'love, sweetheart' via $/ 2 /$-mutation into $/ \mathrm{k} /$; kaa3ib, kawaa3ib (pl.) 'a cuplike-breasted girl, a young beautiful girl' via /3/-loss (cf. cube/cubic from Arabic $k a 3 b$ 'a cube' via /3/-loss); or qa2b, qu2baan (pl.) 'prostitute' via $/ 2 /-$ loss.

Consort (consortium) via Latin consortem, consors 'wife, brother, sister; partner' as a combination of (i) conabove and (ii) sors 'a share, lot' from Arabic shareek 'partner', shirk/sharaaka(t) (n) 'a share' where /sh \& k/ became /s/ both; Sihr 'in-law' in which /S \& h/ merged into /s/; sariat 'wife, girlfriend', sirr 'sex, marriage, secret'; or qaaSirat 'wife, daughter', merging /q \& S/ into $/ \mathrm{s} /$.

Condemn (condemnation, damnation) from Arabic dhamm 'scorn' where /dh/ became /d/. See damn.

Contempt (contemptible, contemptuous) via Latin contemnere 'to slight, scorn' as a combination of (i) con- above and (ii) temnere 'scorn' from Arabic dhamm 'scorn' where /dh \& m/ became /t \& n/.

Copulate (copulation) from Arabic 2abal, ta2beel (n) 'to impregnate', replacing / $2 /$ by /k/; or qabbal, qubla(t) (n) 'to kiss' via lexical shift and /q/-mutation into /k/ (cf. cable from Arabic $2 a b l$ 'cable, rope' where $/ 2 /$ became $/ \mathrm{k} /)$.

Cordial (cordiality, cardiac, cardiology; heart) via Latin cordialis 'of the heart', cordis 'gen.', cor 'heart' from Arabic Sadr 'heart' via reordering and turning /S/ into /k/ (Jassem 2013h). See heart.

Couple (coupling) from Arabic qabeel(aan) 'companion, rival'; /q/ was replaced by $/ \mathrm{k} /$.

Court (courtship) from Arabic gharrad 'sing', changing /gh \& d/ to /k \& t/; zaghrad 'to sing, to trill', merging /z \& $\mathrm{gh} /$ into $/ \mathrm{k} /$ and turning /d/ into $/ \mathrm{t} /$; or $\operatorname{qar}(D / D h)$ 'to praise', replacing /q \& D/ by /k \& t/.

Covet (covetous) from Arabic shahwat 'desire', substituting $/ \mathrm{k} \& \mathrm{v} /$ for $/ \mathrm{sh} \& \mathrm{~h} /$.

Coy from Arabic 2ayaa', 2ayi (adj.) 'shy'; /2/ turned into /k/. See shy.

Crave from Arabic raghiba 'desire' via reordering and changing /gh \& b/ into / $\mathrm{k} \& \mathrm{v} /$.

Cuddle from Arabic 2aDan 'cuddle'; 2 \& n/ became /k \& 1/.

Cunt from Arabic 2inn/hinn 'vagina', 2annat 'wife', 2an2an (v) 'to be horny', turning $/ 2 /$ into $/ \mathrm{k} /$ and inserting $/ \mathrm{t} /$.

Cupid (cupidity) via Latin cupere 'to love' from Arabic $2 u b b$, 2 abeeb(at) 'love, beloved (female)'; /2/ evolved into /k/.

Curse (accurse, cuss) from Arabic rij(s/z) 'dirt' via reordering and turning $/ \mathrm{j} /$ into $/ \mathrm{k} /$; or $k h a(s / z) i^{\prime} a$ 'to cuss, to accurse' where $/ \mathrm{kh} /$ became $/ \mathrm{s} /$ and $/ \mathrm{r} /$ was inserted.

Cuss from Arabic $k h a(s / z) i^{\prime} a$ 'to cuss, accurse'; /kh/ became $/ \mathrm{s} /$.

Cute from Arabic kaiyes/kaiyet 'clever, nice', ghaD 'soft, fresh' by turning /gh \& $\mathrm{D} /$ into / $\&$ t/, or dhaki 'clever' via reversal and replacing $/ \mathrm{dh} /$ by $/ \mathrm{t} /$.

Dally (dalliance) from Arabic dala3 'dalliance, loving treatment' or dalah 'love', deleting /3 \& h/.

Damn (damnation, condemnation) from Arabic dhamm 'scorn' where /dh \& m/ became /d \& n/. See condemn.

Dandy-Andy See Andrew.

Date from Arabic qawaad 'a date, pimp' via reordering and turning /q \& d/ into /d \& t/ or daiooth 'pimp', changing /th/ to /t/; waqt 'time, date' or qoot 'food: dates, wheat' via reordering and replacing /q/ by /t/ (see Jassem $2013 c, j)$.

Darling See dear.

Dear (endear, endearment, darling) from Arabic dalaal 'endearment' or dalah 'to go mad in love'; /r/ replaced /1/ and $/ \mathrm{h} /$ was deleted.

Desire from Arabic sharah, sharaaha(t) 'strong desire', merging /sh \& h/ into /s/ or suroor, tasria(t), sarra (v) 'happiness'.

Despise (despicable) via French despire and Latin despicere 'look down on' of (i) de 'down' from Arabic ta2t 'down, under' via $/ 2 /$-loss and merging $/ \mathrm{t} \& \mathrm{t} /$ into /d/ and (ii) sp(i/e)cere 'look at' from Arabic baSS/baSbaS, tabaSbuS (n) 'look' via lexical shift, reordering, and turning /t \& S/ into /d \& s/ or shaba2a 'look' where /sh $\& 2 /$ became /s/ (Jassem 2013o) (cf. sabba, sabsab(at) (n), tasabsub (n) 'swear at, insult'). 
Detest (detestation) from Arabic Sadad 'hate, turn away' or taDaad 'hate, opposition' via reordering and turning /S \& d/ into /s \& t/ (cf. Jassem 2012i).

Disgusting (gusto) via French degout and Latin gustus, gustare (v) 'taste' from Arabic qashsha(t), taqsheesh (n) 'eating', turning /q \& sh/ into g \& s/; dhawq(at) 'taste' via reversal and turning /dh \& q/ into /st \& g/; or qazzaz, qizz(at)/taqazzuz (n) 'to disgust' where /q \& z/ became $/ \mathrm{s} \& \mathrm{t} /$.

Eager (eagerness) from Arabic 2urqa(t) 'eagerness, a burning (feeling)' via reordering and $/ 2 /$-loss; or 'ariq 'be unable to sleep' via reordering and replacing /q/ by /g/.

Earnest from Arabic anees(at) 'nice, kind'; /r/ split from /n/.

Ejaculate (ejaculation) via Latin eiaculatus, eiaculari (v) 'throw out' of (i) ex 'out' from Arabic aqSa/qaaSi 'out, far' via /q \& S/-mutation into / $\mathrm{k} \& \mathrm{~s} /$ and (ii) iaculari, iacere 'throw' from Arabic jara/sharra/kharra 'run, flow, leak' where /j (sh)/ became /s (k)/; shakhkha 'urinate', turning /sh \& kh/ into /j \& k/; or shakhal(at), tashkheel (n) 'to drip, sieve' where /sh \& kh/ became /j $\& \mathrm{k} /$ (Jassem 2013h).

Elegant (elegance) via Latin elegantia 'taste, refinement, propriety', eligere (v) 'choose' from Arabic 'alaqa, ta'allaq 'to shine' or 'anaaqat 'elegance' via reordering and /q/-mutation into /g/.

Embrace from Arabic raqaba(t) 'neck; a rounding' via lexical shift, reordering, and turning /q/ into /s/ (Jassem 2013h, o).

Emotion (emotive, move) via Latin emovere 'move out, remove, agitate' of (i) ex 'out' from Arabic aqSa/qaaSi 'out, far' via /q \& S/-mutation into $/ \mathrm{k} \& \mathrm{~s} / \mathrm{and}$ (ii) movere 'move' from Arabic math3 'a woman's walk', turning /th \& 3/ into /t \& Ø/ or merging them into /v/; madda 'go out, extend', maDa 'go' or maTTa (tamaTTAT) 'extend' where /d (T)/ became /t/ (Jassem 2013n); damm 'lit. blood; emotion' via lexical shift, reversal, and turning /d/ into $/ \mathrm{t}(\mathrm{v}) /$; or mas $3 / m a 2 t$ 'strong sex' via lexical shift and merging /s \& 3 (2)/ into /t (v)/; or miqat, wamaq 'love', wamaqa (v) where /q \& $\mathrm{t} /$ merged.

Enemy (enmity) via Latin inimicus 'enemy' of (i) in 'not' from Arabic in 'not' (Jassem 2013b) + (ii) amicus 'friend', amare (v) 'to love' from Arabic haama 'to love madly', deleting /h/; or raama 'to love' via reversal. See amicable.

Enniouky via Dutch for 'making love' from Arabic naak, naik/niaka(t) (n) 'to make love, fuck'. See know.

Enthrall (enthrallment) from Arabic dhalla/indhalla, dhaleel (adj.) 'to fear, to be humbled', turning /1/ into /r/; or 'asara, in'asara 'captivate' where /s/ developed into /th/ and /1/ split from /r/.

Entice from Arabic dhaaq, yindhaaq 'to taste' via lexical shift and turning /dh \& q/into /t \& s/.

Erect (erection) from Arabic raaqi(at) 'high', raqa/irtaqa (v) 'to climb'; /q/ became /k/.

Erotic (eroticism, Eros, Erasmus) via Greek eros, erates (pl.) 'lit., love; sex' from Arabic 'arr 'making love', 'air 'penis' (Jassem 2013h); or 3urs 'sex, wedding' via /3/-loss.

Excite (excitement, incite) from Arabic shaaTa, istashaaTa 'excite, to get excited', turning /sh \& T/ into /s \& t/.

Eunuch via Latin eunuchus 'castrated slave' from Arabic nakhs 'slave' via $/ \mathrm{kh} \& \mathrm{~s} /$-merger into $/ \mathrm{k} /$.

Faith (faithful) from Arabic 3afaaf, 3iffat 'chastity'; /3 \& f/ became $/ \varnothing \& \mathrm{th} /$.

Fancy (fantasy, fantasize, fantastic) via Greek phantasia 'image, perception, appearance', phos 'light' from Arabic baSeeS 'small light' where /b \& S/ became /f \& s/; nafs, nafsi 'self, my self; I like/fancy' via reordering; fatana 'astonish, amaze, seduce', faatin (adj) 'beautiful, fantastic' via reordering and turning /t/ into /s/ (Jassem 2013p).

Feminine (femininity, female) via Latin femina 'female' and French femme 'woman' from Arabic untha 'female, feminine' via reordering, /th/-mutation into /f/, and $/ \mathrm{m} /$-split from /n/. See Jassem (2013h, k).

Fertile (fertility, fruit) from Arabic thamar(at) 'fruit' via reordering and merging /th $\& \mathrm{~m} /$ into /f/.

Fiancé from Arabic funuq 'a young, big, beautiful, luxurious, good-mannered girl; luxury', replacing /q/ by /s/; or nufasaa' 'a newly-delivered woman' via reordering.

Fidelity (Fidel; infidelity) from Arabic faDeelat, faDeel/faaDil (adj) 'virtue, fidelity'; /D/ became /d/.

Fiend (fiendish) via Old English feond 'enemy', feogan (v) 'to hate' from Arabic kaafa2 'attack' via reversal, turning $/ \mathrm{k} /$ into $/ \mathrm{g}(\mathrm{ie}) /$, and $/ 2 /$-loss.

Fine from Arabic zain 'fine, beautiful'; /z/ became /f/. See nice.

Foe via Old English gefan 'to hate' from Arabic faa3i, af $3 a$ 'angry, evil man; snake' via reordering and turning /3/ into $/ \mathrm{g}(\varnothing) /$.

Fond (fondness) 'foolish, silly; dote upon' from Arabic fanad 'to dote upon, lie, grow old, to go mad in love, to refuse'.

Fondle (fond) frequentative of fond'. (Cf. Arabic dandal 'suspend, draw out (one's tongue)' where /d/ became /f/.)

Foreplay via (i) Latin pre- 'before' from Arabic (i) $q a b l$ 'before' where /q \& r/ merged into /r/ and /b/ became /f/ and (ii) Old English plegan 'play' from Arabic la3ib 'play' via reordering and turning /3/ into /g (y)/ or (ii) bi3aal 'intercourse', baa3al 'to play with one's wife' via /3/-loss. See playful.

Fornication via Latin fornicari, fornix 'a brothel; arched, vaulted chamber' from Arabic fareesh 'a sex slave girl', farsh, mafaarish (pl.) 'women; furniture; spreading', farasha, iftarasha, infarasha (v) 'to make love with a woman lying on her back', turning $/ \mathrm{sh} /$ into $/ \mathrm{k} /$ and splitting /n/ from /r/; farnasat 'a woman's good house management' via lexical shift and turning /s/ into $/ \mathrm{k} /$; faarin(at) '(female) baker; big man/dog', furn (n) 'oven, bakery' via lexical shift and turning $/ \mathrm{t} /$ into $/ \mathrm{k} /$. 
Frigid (frigidity, fridge, refrigerate) from Arabic thalj 'snow'; /th \& 1/ became /f \& r/ (Jassem 2013d).

Frolic via Dutch vrolyc 'happy' of vro 'merry, glad' and lyc 'like' and German fröhlich 'happy' from Arabic fari2 'happy' where /2/ was dropped or became /h/ in German; or fahr, ifhaar 'sex' via reordering or $/ \mathrm{h} /$-loss.

Frown from Arabic nafar 'frown, come up, bulge' via reordering.

Fuck from Arabic wiqaa3, wa(a)qa3 (v) 'intercourse' via /w \& q/-mutation into /f \& k/ and /3/-loss; hack/haqq 'intercourse' where /h/ became /f/; fakhfakh 'to have a long fuck' via syllable merger and turning $/ \mathrm{kh} /$ into $/ \mathrm{k} /$, baak 'donkey mating', turning /b/ into /f/; fakhat 'deflower, rip', merging $/ \mathrm{kh} \& \mathrm{t} /$ into $/ \mathrm{k} /$; or sifaa 2 'adultery, illegal sex' via $/ \mathrm{s} \& \mathrm{f} /$-merger and $/ 2 /$-mutation into $/ \mathrm{k} /$.

Gay (gaiety) from Arabic ghai, ghiwaiat (n) 'gay, proud', turning /gh/ into /g/.

-Gamy (polygamy, monogamy) from Arabic jimaa3 'sex, intercourse, marriage, joining' via $/ 3 /$-loss and replacing $/ \mathrm{j} /$ by $/ \mathrm{g} /$; or kaama3 'make love', kim3/kamee3 (n) 'husband' via /3/-loss and $/ \mathrm{k} /$-replacement by $/ \mathrm{g} /$.

Glad from Arabic ragheed, raghd (n.) 'happy' via reordering and turning /gh \& $\mathrm{r} /$ into $/ \mathrm{g} \& \mathrm{l} /$.

Good (goodness) from Arabic jood, jayed, jawaad 'good, kind, generous'; /j/ turned into /g/.

Grope from Arabic qaDab 'catch'; /q \& D/ became /g \& r/.

Guilt (guilty) from Arabic ghalaT 'wrong', turning /T/ into $/ \mathrm{t} /$. See regret.

Hanky-panky (hunk) from Arabic hinagh 'a playful, funny woman; adulteress', haanagh (v) 'to court' where /gh/ became $/ \mathrm{k} /$ and baaq 'the sound of making love' via /n/-insertion; or khaaq baaq 'the sound of having sex', turning $/ \mathrm{kh} \& \mathrm{q} /$ into $/ \mathrm{h} \& \mathrm{k} /$ and inserting $/ \mathrm{n} /$.

Harlot from Arabic 3aahirat 'whore', turning /3/ into /w/ and inserting $/ 1 /$. See whore.

Harass (harassment) from Arabic 2arasha, ta2arrash 'harass, aggress'; /2 \& sh/ became /h \& s/.

Hard Core from Arabic (i) 3atr 'hard' via reordering and turning $/ 3 \& \mathrm{t} /$ into $/ \mathrm{h} \& \mathrm{~d} /$ and (ii) qa3ira(t) 'oversexed woman', qu3air 'inner vagina, sex', qa3r 'innermost bottom, depth' where /q \& 3/ became /k \& Ø/ (Jassem 2013c).

Hate from Arabic 2aqida, 2iqd (n) 'hate'; /2/ became /h/ and /q \& d/ merged into /t/; or hatta 'to be disrespectful to, to dishonour'.

Have (haben in German) from Arabic wahab 'give' or $h a b a / 2 a b a$ 'give' via lexical shift, merging /w \& h/, and turning $/ \mathrm{b} /$ into $/ \mathrm{v} /$.

Heart (sweetheart, cordiality, cardiac, cardiology) via Latin cordis 'gen.', cor 'heart' from Arabic Sadr 'heart, breast' via reordering and turning $/ \mathrm{S} \& \mathrm{~d} /$ into $/ \mathrm{h} \& \mathrm{t} /$ (Jassem 2013h). See cordial.

Hooker from Arabic 2aariq(at) 'a woman with a tight vagina; strong sex' via reordering and turning $/ 2 \& \mathrm{q} /$ into $/ \mathrm{h} \& \mathrm{k} /$.
Honour (honourable, honorific) from Arabic 2aram, 2urma(t) 'honour, prohibition' or karam 'honour, generosity' via reordering and turning $/ 2(\mathrm{k}) \& \mathrm{~m} /$ into $/ \mathrm{h} \& \mathrm{n} /$.

Houri from Arabic 2oor(i) 'beautiful, white'; /2/ turned into $/ \mathrm{h} /$.

Hope (hobby) from Arabic $2 u b b(i)$ 'love, hope'; /2/ became $/ \mathrm{h} /$.

Horny from Arabic qarn 'horn', qarnaa' 'horny; a woman with a vaginal in-growth or protrusion, hampering full sex' where /q/ became /h/; or haram, mahroom (adj.) 'oversexed, horny', turning $/ \mathrm{m} /$ into $/ \mathrm{n} /$.

Hospitable (hospice, hospital) from Arabic 3izba(t), $3 a z z a b$ (v) '(offering) food and drink', turning $/ 3 \& \mathrm{z} /$ into $/ \mathrm{h} \&$ $\mathrm{s} /$.

Hostile (hostility, host) via Latin hostis 'enemy' from Arabic haash, hawshat (n) 'attack, fight', hawwaash(at) (n) 'fighter(s)' where /sh/ became /s/ (Jassem 2013p); or jaish 'army' in which $/ \mathrm{j} \& \mathrm{sh} /$ became $/ \mathrm{h} \& \mathrm{~s} /$.

Hug from Arabic $2 a(w) a q$ 'encircle; reach something high; centre', turning $/ 2 \& \mathrm{q} /$ into /h \& g/; hack/haqq 'strong sex' via lexical shift and replacing /k (q)/ by /g/; 3aanaq 'embrace', 3unug (n) 'neck', turning /3 \& q/ into /h \& g/ and deleting /n/; shawq 'longing' or shiq 'breast, side, section', turning /sh \& q/ into /h \& g/.

Hunk (hanky-panky) from Arabic hinagh 'a playful, funny woman; adulteress', haanagh (v) 'to court'; /gh/ became $/ \mathrm{k} /$.

Husband from Arabic 2aseeb 'relative, noble, husband', replacing / $2 /$ by /h/ (Jassem 2013k).

Ill-behaved (behave, behaviour) from Arabic (i) la 'not, ill-' via reversal (Jassem 2013b) or 3aleel 'ill, sick' via /3/-loss and (ii) hadhdhaba 'to behave, make polite' via reordering and turning /dh/ into /v/ or 'adab 'good manners, politeness' via reordering and changing /', $\mathrm{d}$, $\& \mathrm{~b} /$ into $/ \mathrm{h}, \mathrm{dh}, \& \mathrm{v} /$.

Impotent (potent, potency, potential) via Latin impotens of (i) in/(im) 'not' form Arabic in 'not' (Jassem 2013b) + (ii) potens 'power, control', potere (v) from Arabic baaDa3, mubaaDa3a(t) (n) 'to make love' via /3/-loss or mutation into /n/ and replacing /D/ by /t/.

Impregnate (pregnant) via Latin impregnare 'to make pregnant' from Arabic ibraak 'make love on the side or on all fours', turning /k/ into /g/; abraqat 'of a woman, to get ready for sex' (cf. Jassem 2013k).

Incest (chastity) via Latin incastus 'relative sex' of (i) in 'not' from Arabic in 'not' (Jassem 2013b) + (ii) castus 'pure; cut-off, separated' from Arabic qazaazat 'shyness, modesty', $q(a / i) z z(a t)$ 'a nice, modest person, who shuns blemishes' via lexical shift and /q \& z/-mutation into /s/ both. (Cf. qashaT/kashaT 'to strip, separate' where /q \& sh/ changed to /s/.) See chaste.

Infatuate (infatuation) via Latin fatuus 'foolish person', infatuare (v) 'bring something to foolishness' from Arabic fadad/fadfad, fadeed (n) 'to shout; to walk proudly', faddaad (n) 'a rough and tough farmer; coward; loud-spoken person', turning /d/ into $/ \mathrm{t} /$; or 
fatan, infatan 'infatuate'.

Infidelity (infidel) See fidelity.

Insult from Arabic salaT, saleeT (adj.) 'bad talk'; /T/ became $/ \mathrm{t} /$.

Intercourse from Arabic 3irs, 3arras (v) 'wedding, making love' where $/ 3$ / evolved into $/ \mathrm{k} /$; or kharoos 'a virgin in early intercourse', turning $/ \mathrm{kh} /$ into $/ \mathrm{k} /$.

Inviting (invitation, invite) via French invité 'guest' from Arabic Daif, inDaaf(v) 'guest' via reversal and turning /D/ into / $/$ /

Irony (ironical) from Arabic nahara 'to shout one down' via reordering and /h/-loss (Jassem 2013i).

Joy (enjoy, rejoice) from Arabic jawa 'internal love' via lexical shift or zahoo 'joy, brightness' via /z \& $\mathrm{h} /$-merger into $/ \mathrm{j} /$.

Keen (keenness) from Arabic 2an(een) 'longing, yearning'; $/ 2 /$ became $/ \mathrm{k} /$.

Kill (killer) from Arabic qatal 'kill' where /q \& t/ merged into /k/ or ghaala 'kill' in which /gh/ became /k/.

Kind (kindness) from Arabic 2inniat 'kindness, longing, yearning', 2anoona(t), 2aanna(t) (adj.) /k \& d/ replaced $12 \& \mathrm{t} /$.

Kiss from Arabic $k a(a) f a 2$, kifaa2 (n) 'kiss suddenly', merging /f \& 2/ into /s/; kass 'of teeth, short' or kazza 'to press/push (teeth)' via lexical shift and changing / $\mathrm{z} /$ to /s/ (Jasem 2013o).

Know 'make love' from Arabic naak 'make love' via reversal; aiqan 'know' (Jassem 2013p); or 3alima/a3lam 'know', $3 i l m(\mathrm{n})$ via reordering, turning $/ 3 /$ into $/ \mathrm{k} /$, and merging $/ 1 \& \mathrm{~m} /$ into $/ \mathrm{n} /$ (cf. acknowledge).

Lascivious via Latin lascivia 'lewdness, playfulness, frolicsomeness' from Arabic lahoo 'playfulness', turning $/ \mathrm{h} \& \mathrm{w} /$ into $/ \mathrm{sh} \& \mathrm{v} /$.

Lechery (lecherous, lick) 'lit., licking' from Arabic la2s/la3q 'licking, eating' where /2 (3) \& s (q)/ merged into /ch/ or laqq/laqlaq 'of dogs, to tongue-drink' where /q/ became $/ \mathrm{ch} /$. See luxurious.

Lesbian (lesbianism. lesbo) via Latin/Greek lesbi(u/o)s 'relating to the Greek Island of Lesbos 'wooded?' from Arabic laSb 'a mountain gap; a valley course; of flesh, to stick to bones due to impoverishment', lasb 'sting, stick, lick' via lexical shift, or lazb 'narrow road; sting; stick; enter into; drought; a collocate of $3 a z b$ lazb 'unmarried' via lexical shift; salhaba(t) 'a big woman with little flesh on' via reordering and /h/-loss; shabal 'of male sheep, mounting/mating' via lexical shift, reordering, and changing $/ \mathrm{sh} /$ to $/ \mathrm{s} /$.

Lewd from Arabic looTi 'homosexual', turning /T/ into /d/; la3aT 'to eat quickly', deleting $/ 3 /$ and changing $/ \mathrm{T} /$ to /d/; or laT3 $\left(a^{\prime}\right)$ 'a vagina with little flesh on; stiff vagina' via lexical shift, /3/-loss, and turning /T/ into /d/.

Lie via Old English legan from Arabic aliqa, waliqa 'lie, say an untruth' or laqa2 'lie (on bed)', turning /q \& 2/ into /g (Ø) \& Ø/ (Jassem 2013i, n).

Like from Arabic aliqa 'love, similar' or laaqa 'be suitable to, like', turning /q/ into /k/; or qala 'hate' via lexical divergence and reversal.
Lip from Arabic laabba(t), labaaleeb (pl.) 'lip, loving'.

Loath (loathe) via Old English lathian, lath (adj) 'hate, hostile' from Arabic ludd, ladood 'hostile'; /d/ turned into /th/.

Long (longing) from Arabic law3(an)/lawj 'love' via $/ 3$ (j)/-mutation into $/ \mathrm{g} /$; tawq(aan) 'long for' via reordering and turning /t \& q/ into / \& g/; or 3alaaqa(t) 'love' via reordering and turning $/ 3 \& \mathrm{q} /$ into $/ \mathrm{n} \& \mathrm{~g} /$.

Love (loveable) via Old English lufu, lufian (v) 'love' and German lieben 'love' from Arabic lahaf 'love, strong desire' via /h/-loss, labab, 'alabba, laabba(t) (n) 'to love, to live/stay' where /b/ became /v/; labba, lablab 'of animals, to lick and fondle with lips; to sympathize with' (cf. believe from Arabic aballa, ubaalat (n) 'to become a priest' via reordering).

Lust from Arabic ladhdhat, ladheedh (adj.) 'lust, delicious', turning /dh/ into /s/.

Luxurious (luxury) via Latin luxuria, luxus 'excess, delicacy, non-chastity, lechery' from Arabic la $2 s / l a 3 q$ 'licking, eating' or laqs 'eating', turning $/ 2$ (q)/ into $/ \mathrm{k} /$. See lechery.

Man from Arabic nama, anaam (pl.) 'man' via reversal (cf. German Mensch 'man' from Arabic insaan 'man, human' via reordering and turning $/ \mathrm{n} \& \mathrm{~s} /$ into $/ \mathrm{m} \& \mathrm{sh} /$ (Jassem 2013g).

Marry (marriage, marital, matrimony, matron; mother) via Latin maritus 'husband', mari 'woman', maritare (v) 'to marry' from Arabic mar'a(t) (mara in the vernacular) 'woman' via lexical shift (Jassem 2013k).

Macho (masculine, male) via Spanish from Latin masculus 'male' and French maisle from Arabic zalama(t) 'man' via reordering, /z/-mutation into /ch/, and $/ 1 \&$ m/-merger. See Jassem (2013h, k).

Masturbate (masturbation) from Arabic maSara 'to milk out by pressing nipples'; /S/ split into /st/ and /b/ was inserted.

Mercy (merchant, mercenary, market) via Latin merces 'reward, wages, hire pay', merx 'wares, merchandise', mercare (v) 'to trade' from Arabic mutaajara(t) 'trading' via reordering and merging $/ \mathrm{t} \& \mathrm{j} /$ into $/ \mathrm{s} /$; maSaari 'money' via reordering and lexical shift; ma'joor 'paid, thanked' or mashri 'bought' via reordering and turning /j (sh)/ into /s/; $\operatorname{mar} 2 a$ 'an exclamation of pleasure for achievement; an appreciation; a bravo' where /2/ became /s/ (cf. bar2a 'its antonym' and bravo via divergence); or ra2eem 'merciful' via reordering and turning $/ 2 /$ into $/ \mathrm{s} /$.

Merry (merriment) from Arabic mari2 'happy'; /2/ was dropped.

Miser (miserable, miserliness) from Arabic mu3aththar 'miserable, poor' via /3/-loss and turning /th/ into /s/ or mu3sir 'needy, one in difficulty' via $/ 3 /$-loss.

Modest (modesty) via Latin modestus 'modest', modestia 'moderation, honour, good conduct', modus 'measure, manner' from Arabic muTaiTa', maTTa (v) 'arrogant walking' via lexical shift or divergence and changing /T/ to /d/; mataa3 'vagina, pleasure', mut3a(t) 'pleasure 
(convenience marriage)' via lexical shift, turning /t \& 3/ into $/ \mathrm{d} \& \mathrm{~s} /$; or muTee3(at) 'obedient, modest' where $/ \mathrm{T}$ \& 3 / became /d \& s/.

Moral (morality, more) from Arabic muru'a(t) 'morality, knowledge, power'.

Naked from Arabic na2a 'take off (clothes)', replacing / / by $/ \mathrm{k} /$; or naqi(at) 'pure' via lexical shift and turning /q/ into $/ \mathrm{k} /$.

Nice (nicety) from Arabic zain(at) 'nice, beautiful' or 2asan 'nice'; reversal, turning /z/ into /s/, and merging /2 \& s/ applied.

Noble (nobility) from Arabic nabeel 'noble, respectable, intelligent' (Jassem 2013p).

Nude (nudity) from Arabic naDa, naDu (n) 'take off clothes'; $/ \mathrm{D} /$ turned into $/ \mathrm{d} /$.

Odd (oddity) from Arabic 3adoo, 3adawat 'enemy, enmity' via /3/-loss or waa2id, a2ad 'one' via /2/-loss (Jassem 2012a).

Oral (orifice) from Arabic thaghr 'mouth' via /gh \& $\mathrm{r} /$-merger into / $\mathrm{r} /$ or furja $(t)$ 'an opening' via reordering and replacing /j/ by /s/ (Jassem 2013h); or ghaar/ghawr 'cavity' via /gh \& r/-merger.

Organ from Arabic 3irq, 3urooq (pl.) 'vein, organ' or 3arq 'a bone with little flesh on' via /3/-loss and /n/-insertion.

Orgasm via Latin/Greek $\operatorname{orgasm}(u s / a)$ 'excitement, swelling', $\operatorname{organ}(\mathrm{v})$ 'to be on heat, swell, become ripe for' from Arabic qarn 'horn, to bulge like one; horny', 2araqaan, $2 \operatorname{araq}(\mathrm{v})$ 'burn', 2aariqa(t) 'a woman with a tight (burning) vagina' via $/ 2 /$-loss and changing /q/ to /g/; haraj 'sex' via /h/-loss and turning/j/ into /g/; rahaz 'to sexually shake and move', turning $/ \mathrm{h} /$ into $/ \mathrm{g} /$ or ra3ash(an) 'shivering' via $/ 3, \mathrm{sh}, \& \mathrm{n} /$-mutation into /g, $\mathrm{s}, \& \mathrm{~m} /$; or 2 arqaS 'sit uncomfortably as in restraining the call of nature' via /2/-loss and turning /q \& S/ into /g \& s/.

Passion (passionate) via Latin passio(nem), pati (v) 'suffering, enduring' from Arabic $b u$ 's 'miserliness, suffering', ta3ab 'tiredness' via reordering, lexical shift, and $/ 3 /$-loss, or 3adhaab 'suffering' via reordering, /3/-loss, and turning /dh/ into /t (s)/.

Pathos (pathetic, sympathy) via Greek pathos 'sorrow' from Arabic bathth 'extreme sorrow'.

Penis 'tail in Latin' from Arabic dhanab 'tail' via reversal and turning /dh/ into /s/ (Jassem 2013h).

Philo (philanthropy) from Arabic balah, walah 'love', turning /b (w) \& h/ into /f \& Ø/ or labab/labba 'love' via reversal and turning $/ \mathrm{b} /$ into $/ \mathrm{f} /$.

Phyllis (phallic) from Arabic faishala(t) 'penis head' via reordering and turning /sh/ into /s/ (Jassem 2013h).

Piss-off from Arabic zubb-fee 'penis in- a swearing' via reversal and turning /z/ into /s/ or baz(baz), bas(bas) 'flow, pass, come out'; azib 'run, flow' via reordering and changing /z/ to /s/; or bashsh 'of babies, urinate', turning /sh/ into /s/ (Jassem 2013d, h).

Playful from Arabic ba3l 'wife, husband', bi3aal 'intercourse', baa3al (v) 'play with one's wife' via /3/-loss. See foreplay.
Please (pleasure, pleasant) from Arabic bajal 'to praise, to bring joy' via reordering and turning $/ \mathrm{j} /$ into $/ \mathrm{s} /$ or bashshar 'to make happy' via reordering and turning /sh \& r/ into /s \& 1/ (Jassem 2013i).

Polygamy (polygamous) See -gamy.

Pornography via Greek porne 'lit., purchased; prostitute, a female slave bought for sex' from Arabic (i) rabeeb, rabbat, rubban 'a slave' via reordering, rabaab 'a woman in recent delivery' or zanboor 'clitoris' via lexical shift, reordering, and merging $/ \mathrm{z} \& \mathrm{~b} /$ and (ii) 2 arf 'letter, writing' where /2/ became /g/ (Jassem 2013i).

Pretty from Arabic barTa(t) 'beautiful' where /T/ turned into /t/; or barahraha(t) 'very beautiful/white' via /h/-loss.

Prostitute (prostitution) via Latin prostitus from (i) pro 'before' from Arabic qabl 'before' via reordering and merging /q \& 1/ into /r/ and statuere 'cause to stand' from Arabic saTa2a, saT2at (n) or sada2a, sad2at (n) 'to lie one ones back' via /2/-loss.

Pride (proud) from Arabic baTar 'pride, evil' via reordering and turning $/ \mathrm{T} /$ into $/ \mathrm{d} /$ (cf. bride.)

Pure (purity, puritan) from Arabic barahraha(t) 'very white' via /h/-loss, baree', baraa'a(t) (n) 'innocent', or baarr 'fair, kind'.

Pussy from Arabic biss(at) 'cat' or $z u b b$ 'penis' via lexical shift or divergence, reversal, and turning /z/ into $/ \mathrm{s} /$.

Queen (gyne-) from Arabic qain(at) 'beautiful woman, singer' via lexical shift.

Ram from Arabic ghanam 'sheep' where /gh \& n/ merged into /r/ or qa2am 'ram, advance, push ahead' in which /q \& $2 /$ became $/ \mathrm{r} \& \varnothing /$.

Rape (rapist) from Arabic 'irb 'intercourse, vagina' via lexical shift (cf. pray from Arabic 'ariba 'prostrate, pray, fall' via reordering).

Rascal from Arabic radheel 'lowly, base' where /dh/ split into $/ \mathrm{s} \& \mathrm{k} /$.

Regret (regretful) from Arabic ghalaT 'wrong' via lexical shift and turning /gh \& $1 /$ into $/ g \& \mathrm{r} /$. See guilt.

Revile (vile) via Latin vilis 'cheap, worthless, base' from Arabic dhaleel, dhill/dhilla(t) (n) 'lowly, frightened' or radheel 'lowly, base'; /dh/ became /v/.

Romantic (romance, Rome, Roman) via Latin Rome 'a fortified enclosure' from Arabic 'araam 'rocks', raama, ra'oom (adj.) 'to love', or gharaam, gharmaan 'love', turning /gh/ into /r/.

Rude from Arabic radee' 'bad, rude'.

Rue (rueful) from Arabic rawia(t), tarawwi 'thoughtfulness' via lexical shift.

Sarcasm (sarcastic) from Arabic sukhria(t), maskhara(t), sakhira (v) 'sarcasm' via reordering and replacing $/ \mathrm{kh} /$ by $/ \mathrm{k} /$.

Satire (satirical) from Arabic izdara 'despise, look down upon'; /z \& d/ were replaced by /s \& t/.

Scorn from Arabic zajar, zujraan (n) 'scorn' where /z \& j/ were replaced by /s \& k/ or 2aqara, 2uqraan (n) 'look down on' in which $/ 2 \& \mathrm{q} /$ became $/ \mathrm{s} \& \mathrm{k} /$.

Seductive (seduction, seduce, deduce, deduct, conduce, 
reduce, produce, educate, duke) via Latin seducere 'lead away/astray' from (i) se 'away, aside' from Arabic zaa 2 'shift, to be away' via /z \& 2/-merger into /s/ (see Jassem 2013a-b) and ducere 'lead to' from Arabic qaada, qiadat (n) 'lead' via reordering and replacing /q/ by /s (k)/ (Jassem 2013i, p).

Semen (insemination) from Arabic samn, sumna(t)/tasmeen (n) 'fat, butter' via lexical shift; mani 'semen' via /s/-insertion or madhi 'pre-semen' via reordering, turning /dh/ into /s/, and /n/-insertion (Jassem 2013h).

Sensation (sense, sensitivity, sensible) from Arabic 2iss, in2ass, ma2soos (adj) 'sense, feeling, voice', turning /2/ into /s/ and inserting /n/ (Jassem 2013o-p).

Sex (sexuality, section) via Latin sexus 'male/female', secare (v) 'divide, cut', seco 'half' from Arabic shaqqa 'cut' where /sh \& q/ became /s \& k/; shakhS 'person' where /sh, kh, \& S/ evolved into /s, k, \& s/; or kuss, kaskas (v) 'vagina' via lexical shift and reordering.

Shame (ashamed, shameful) from Arabic shu'm 'shame'; /'/ dropped.

Shiver from Arabic ra3ash or rajaf 'shiver' via reversal and turning $/ 3 \& \mathrm{j} /$ into /v \& sh/.

Shy from Arabic 2ayaa' (n), 2ayi (adj.), ista2a (v) 'coy, shy'; $/ 2 /$ turned into $/ \mathrm{sh} /$. See coy.

Sleep (schlaffen in German) from Arabic labs 'confusion, sleep' or libs 'wearing, dressing' via reordering and lexical shift.

Slut from Arabic saleeTa(t) 'a rude woman' or shaleeTa 'a slut', turning /sh \& $\mathrm{T} /$ into /s \& $\mathrm{t} /$.

Smart from Arabic amrad 'hairless (male), beautiful' where /d/ turned into /t/ and /s/ was inserted; samt 'clever' or zameet 'clever' via/r/-insertion; amzaT 'thin, slim' via reordering and changing $/ \mathrm{z} /$ to $/ \mathrm{s} /$; zamraT 'thin, beautiful' where /z/ became /s/; maraT 'beautify' via /T/-split into /s \& $\mathrm{t} /$.

Sod (sodomy) via Latin sodomia from Arabic sadam 'love-cum-regret and sorrow; gloom and regret; a male camel' (cf. Surm 'anus; firmness; tightening', turning /r/ into $/ \mathrm{d} /$ ).

Sorrow (sorry) from Arabic za3al 'sorrow', turning /z \& 3/ into /s \& w/; or 2asra(t), 2aseer (adj.) 'sorrow' via $/ 2 \&$ $\mathrm{s} /$-merger.

Sterile (sterility) via Latin sterilis 'barren, unproductive' from Arabic jardaa' 'barren, sterile' via reordering and turning /j \& d/ into /s \& t/; or 3aaqir 'sterile' where $/ 3 \&$ $\mathrm{q} /$ became $/ \mathrm{s} \& \mathrm{t} /$.

Strip Tease via Old English toesan 'pluck, pull apart' from Arabic (i) karab 'pull together, tighten (clothes)' where /k/ split into /s \& t/ and (ii) shadd 'pull, tighten' via reversal and turning /sh \& d/ into /s \& t/; or Darb Teez 'hitting ass' in which /D/ split into /st/ and /T \& z/ became /t \& s/ .

Swear from Arabic shaara, mishaari (n) 'swear at' or 3aiyar, mi3yaar (n) 'swear at'; /sh (3)/ changed to /s/.

Sweetheart (sweetie) from Arabic (i) sitt, sitti 'lady, lady-my (my lady)' and Sadr 'heart, breast = (lady of the heart)' via reordering and changing $/ \mathrm{S} \& \mathrm{~d} /$ to $/ \mathrm{h} \& \mathrm{t} /$. See cordial.

Sympathy (sympathize, apathy) via Latin sympathia from Greek sy $(n / m)$ - 'together' from Arabic jamee 3 'together' via /j/-mutation into /s/ and /3/-loss and pathos 'feeling' above.

Teat from Arabic thadi 'teat', turning / th \& d/ into t/.

Temptation from Arabic taim, mutaiam 'love-enslaved' via $/ \mathrm{p} /$-split from $/ \mathrm{n} /$ ot tabbal, matbool (adj) 'love-sickened' via reordering and turning /1/ into /t/.

Throne (enthronement) from Arabic sareer 'bed, throne'; /s $\& \mathrm{r} /$-evolved into /th \& $\mathrm{r} /$.

Ugly from Arabic qala 'hate'; /q/ turned into /g/.

Upset from Arabic 3absat, 3abas (v) 'frown, anger' via $/ 3 /$-loss or ghaDab 'anger' via reordering and substituting /s \& D/ for /gh \& D/.

Vagina from Arabic farj 'vagina' via reordering and turning $/ \mathrm{r} /$ into $/ \mathrm{n} /$ or 3ijaan 'vagina' where $/ 3 /$ became $/ \mathrm{v} /$.

Valentino (Valentine) 'a good-looking man of carnal desires' from Arabic 2uloo, 2ulwa(t), 2alwaan 'sweet, good-looking (person); marrying a woman for a dowry'; $/ 2 /$ became $/ \mathrm{v} /$.

Vice (vicious) from Arabic fu2sh 'vice', merging $/ 2 \& \mathrm{sh} /$ into /s/; or waSee 'agent, deputy' where /w \& S/ became $/ \mathrm{v} \& \mathrm{~s} /$.

Virgin (virginity, Virgo) from Arabic bikr 'virgin' via reordering. turning $/ \mathrm{b} \& \mathrm{k} /$ into $/ \mathrm{v} \& \mathrm{~g} /$, and inserting $/ \mathrm{n} /$.

Virile (virility) via Latin virilis 'of a man', vir 'man, hero' from Arabic mar' 'man' or 2urr 'hero, free' where /m (2)/ became /v/; fa2l, fu2oola(t) (n) 'virile; oversexed male' via $/ 2 /$-loss and $/ \mathrm{r} /$-split from $/ \mathrm{l}$; or $\mathrm{ba} 3 \mathrm{l}$, bu3oola(t) (n) 'husband, virile' where /3/ became /v/.

Virtue (virtuosity, virtuous) from Arabic $\mathrm{faDl}$ 'virtue, favour' via reordering and turning /D \& $1 /$ into $/ \mathrm{t} \& \mathrm{r} /$ or $3 a r D$ 'virtue, sexual modesty, honour, respect' where $/ 3 /$ turned into $/ \mathrm{v} /$.

Voluptuous via Latin voluptuosus 'full of pleasure, delightful', voluptas 'pleasure, delight, sex' from Arabic wabalat, istawbalat (v) 'of female sheep, a strong need for mating', wabaal 'corruptness' via reordering and turning /w/ into /v/ (cf. khalaabat 'deceptive love', khallaab (adj.) 'beautiful'; or habal, mahbal 'vagina', turning $/ \mathrm{h} /$ into $/ \mathrm{v} /$ ).

Vow (vote) via Latin voiere 'to promise' from Arabic wa' $i$ 'vow, promise; zebra; fast horse'; /w/ became /v/ (Jassem 2013i).

Vulgar (vulgarity) from Arabic faajir 'bad, rude' or faqeer 'poor' where /j \& q/ became /g/ and /1/ split from /r/.

Want from Arabic wadda 'want', mawaddat (n) 'love' via reordering and turning $/ \mathrm{m} \& \mathrm{~d} /$ into $/ \mathrm{n} \& \mathrm{t} /$.

Wanton from Arabic wadan, widaan 'a bride's good treatment', mawaddat 'love' via reordering and turning $/ \mathrm{d} /$ into $/ \mathrm{t} /$.

Wed (wedding) from Arabic wiT' 'making love, marriage'; $/ \mathrm{T} \&$ / were replaced by $/ \mathrm{d} \& \emptyset /$.

Went (in unto) from Arabic faat or afDa 'go in, enter'; /f/ became $/ \mathrm{w} /$ and $/ \mathrm{n} /$ was inserted. 
Whim (whimsical) from Arabic Arabic wahm 'whim, illusion'.

Whore (harlot) from Arabic 3aahir(at) 'whore', substituting /w/ for /3/; rahoo, rahwa 'a whore, woman with a big vagina' via reversal.

Wife from Arabic wafia(t) 'faithful woman' via lexical shift or haifa 'beautiful woman' via lexical shift and changing $/ \mathrm{h} /$ to $/ \mathrm{w} /$.

Wish (wishful) from Arabic mashee'a(t), shaa'a (v) 'wish' via reordering and turning $/ \mathrm{m}\left({ }^{\prime}\right) /$ into $/ \mathrm{w} /$ (cf. wash from Arabic $w u D u$ ' 'wash (for prayer)' where $/ \mathrm{D} /$ turned into /sh/ or maaSa 'wash' in which /m \& S/ became /w $\& \mathrm{sh} /($ Jassem 2013d)).

Woman from Arabic aiyem, aimaan/ayaama (pl.) 'unmarried woman', turning /'/ into /w/; or anaam 'humans' via lexical shift and reordering. See man.

Woo from Arabic hawa 'fall in love with', merging /h \& w/ into $/ \mathrm{w} /$.

Yearn via Old English geornan/giernan, georn (adj.) 'eager, desire' and German gern 'glad', begehren (v) from Arabic gharaam 'love' where /gh \& m/ became /g (y) \& $\mathrm{n} /$ or 2anna, 2aneen (n) 'yearn', turning $/ 2 \& \mathrm{n} /$ into $/ \mathrm{y}$ (g) \& r/.

To sum, the total number of love and sexual words amounted to 239, all of which have true Arabic cognates: i.e., $100 \%$.

\section{Discussion}

The above results clearly indicate that love and sexual words in Arabic, English, German, French, Latin, and Greek are true cognates for manifesting similar or identical forms and meanings. Their differences, however, stem from natural and plausible causes and courses of phonetic, morphological and semantic change. The percentage of shared vocabulary between Arabic and English here was 100\%, which exceeds Cowley's (1997: 172-173) classification according to which an $80 \%$ ratio indicates membership to the same language- i.e., dialects.

In light of the above statements, the results are in harmony with all the findings of previous studies (Jassem 2012a-f, 2013a-p) in which English, German, French, Latin, Greek, Sanskrit and Arabic were all found to be rather dialects of the same language, let alone the same family. Besides, they lend further support to the adequacy of the lexical root theory for the present analysis. The main principle which states that Arabic, English, German, French, and so on are not only genetically related but also are dialects of the same language is, therefore, verifiably sound theoretically and true empirically. The clearest proof to that is tracing English love and sexual words back to true Arabic cognates on all plains of phonetic, morphological, grammatical, and semantic analysis.

On the phonetic plain, the main changes included substitution (assimilation, dissimilation, palatalization, spirantization), reversal, reordering, insertion, split, and merger. On the morphological plain, all affixes had true Arabic cognates (Jassem 2012f, 2013a-b). On the semantic level, lexical stability was the general pattern where words maintained their basic meanings across languages. The recurrence of lexical convergence in the data is due to formal and semantic similarity between Arabic words, on the one hand, and their English cognates, on the other. For example, English love derives from formally and semantically similar Arabic words- namely, lahaf 'love' via /h/-loss or 'alabba (labba) 'love, live/stay' where /b/ became /v/; German lieben/leben 'love/live' (also bleiben 'remain') and Arabic 'alabba are identical cognates. Similarly, semantic multiplicity stems from the same reasons where some English words may have more than one meaning, which may have more than one likely Arabic cognate; for instance, date has three meanings (i.e., time, kind of fruit, lover), all of which derive from formally and semantically similar Arabic words- namely, waqt 'time', qoot 'food', and qawaad 'a pimp' via reordering and/or /q/-mutation into /d/. As can be seen, all are similar in form and meaning. Furthermore, lexical variability was obvious in the use of different words for the same concept such as love/lieben in English/German and amare in Latin and French, both of which exist in Arabic as was shown in the results above. Also lexical shift recurred in many instances such as emotion, hug, kiss. Finally, lexical split occurred as in Arabic $2 u b b$ 'love' which gave Cupid in Latin and hope/hoffen in English/German.

What are the implications of this all? It implies two things at least. The first is that Arabic and English are dialects of the same language for having the same words with similar or identical forms and meanings (cognates), with Arabic being the source or parent language because of its phonetic complexity and lexical multiplicity and variety with over 100 'sex' terms (Altha3alibi 2011: 208) (for detail, see Jassem (2012a-f, 2013a-i). Reverting to the short exemplary love and sexual text in 2.1 above, for instance, the analysis has shown that every single word in it has a true Arabic cognate, which can be checked in the results above and/or the relevant previous studies like Jassem (2012c) for pronouns, (2012d) for determiners, (2012e) for verb 'to be', (2012f) for inflectional morphemes, (2013a) for derivational morphemes, and (2013b) for negative particles. More precisely, the sentence(s)
I hope that you love me.
Ich hoffe, dass du mich liebst.
J'espére que tu m'aimes.

is/are $100 \%$ Arabic, irrespective of the resultant changes.

Secondly, it has interesting implications for general linguistic theory and language origin (Jassem 20131). On the one hand, it implies that the so-called proto-Indo-European language hypothesis is fictitious and baseless which should be rejected outright because all English words, for instance, are traceable to Arabic sources. On the other hand, it implies, on a larger scale, that all human languages are related to one another, which in the end descended from a single 'perfect' source, which suddenly emerged in perfect fashion. Over time, however, it became simpler and simpler like English 
words being simpler than their Arabic cognates phonetically, morphologically, and semantically; today's Arabic words are no exception, which are simpler than Classical Arabic ones. Besides, the change or simplification proceeded extremely slowly over the ages, spanning thousands of years; for example, Pagel et al (2013) found that some 27 common English core words (e.g., pronouns) changed or simplified little during the last $15,000.00$ years!?

Now is it feasible to reconstruct that old, sudden, original, fully-fledged, perfect source, technically known as proto-language (Harper 2012) or proto-world-language (Ruhlen 1987, 1994)? It is, indeed. How? To answer that question more clearly and satisfactorily, a few facts need to be elucidated first about the nature of language acquisition or learning and language change or evolution. As to the former, all human languages are the result of learning; one speaks a language because someone taught him it; it is really impossible, otherwise. Whether it is today's or yesterday's language, in the $21^{\text {st }}$ century or at time zero when humanity first appeared on earth, the same rule applies no matter how long ago that might have been; time is immaterial because the same outcome would still obtain; a million or a billion years make no difference whatsoever. This fact is the axis upon which all first and second language acquisition research worldwide rotates (Crystal 2010; Yule 2006; Jassem 1987, 1993, 1994). Language learning is just like a computer: both the hardware and software have to designed by someone; a computer does not make itself, nor does it make itself run. As for the latter, all languages must have descended, evolved, or originated in the end from one perfect source. Over time, they changed form and meaning but not substance where the essence of the word remained intact. For example, Arabic lahaf 'love' or 'alabba 'love, live', English love/live, and German lieben/leben all kept their substance but changed their form or pronunciation where /b/ became $/ \mathrm{v} /$, for instance. Therefore, in light of these facts, it follows that pre-historic language has remained and survived to this day in contemporary world languages, though in different forms. In other words, all human languages are variable developments of that old, sudden, perfect source; that pre-historic language has never died out completely; it still exists to varying degrees in all human languages in current use.

Which current human language resembles it more closely? This automatically leads one to the question of reconstructing that pre-historic language, which can be successfully achieved on the basis of (an) ancient world language(s), which has survived into modern ones, though in different forms. Of all, Arabic is perhaps the greatest survivor and inheritor, which may be the best possible link to that old perfect language on which analysis should focus. Indeed, Arabic can be said to have maintained almost all the features of that original, perfect language for the reasons adduced above (see Jassem 2012a). Pronouns in world languages is a case in point, which has provided some clues to that (Jassem 2012d, 20131) but more evidence is awaiting further research into the subject.

\section{Conclusion and Recommendations}

The main results here can be summed up as follows:

i) The lexical root theory has been adequate for the analysis of the close genetic relationships between love and sexual words in Arabic, English, German, French, Latin, and Greek according to which they are all dialects of the same language.

ii) The 239 love and sexual words or so in English, German, French, Latin, Greek, and Arabic are true cognates with the same or similar forms and meanings. However, their differences are due to natural and plausible causes and courses or directions of phonetic, morphological, and lexical change (cf. Jassem 2012a-f, 2013a-p).

iii) Phonetically, the main changes included substitution, reversal, reordering, split, and merger; lexically, the recurrent patterns were stability, convergence, multiplicity, shift, split, and variability; the abundance of convergence and multiplicity stem from the formal and semantic similarities between Arabic words from which English and European words emanated in the first place.

iv) The phonetic complexity, huge lexical variety and multiplicity of Arabic love and sexual words compared to those in English and European languages point to their Arabic origin in essence.

v) Finally, further research into all language levels is needed, especially lexis or vocabulary (Jassem 2012a-f, 2013a-p). The application of such findings, moreover, to language teaching, lexicology and lexicography, translation, cultural (including anthropological and historical) awareness, understanding, and heritage is badly needed to promote and disseminate rapprochement and acculturation.

\section{Acknowledgements}

Cordial thanks are warmly extended to everyone who contributed to this research in any way worldwide. For my supportive and inspiring wife, Amandy M. Ibrahim, I remain indebted as ever.

\section{References}

[1] Algeo, J. (2010). The origins and development of the English language. (6th edn.). Wadsworth Cengage Learning.

[2] Altha3aalibi, Abu ManSoor. (2011). Fiqhu allughat wa asraar al3arabiyyat. Ed. by Alayoobi, Dr. Yaseen. Beirut and Saida: Al-Maktabat Al-3aSriyyat.

[3] Bergs, Alexander and Brinton, Laurel (eds). (2012). Handbook of English historical linguistics. Berlin: Walter de Gruyter.

[4] Campbell, L. (2006). Historical linguistics: An introduction. 
(2nd edn). Cambridge, Mass.: The MIT Press.

[5] Celce-Murcia, M. et al. (2010). Teaching pronunciation: A course book and reference guide. (2nd edn). Cambridge: Cambridge University Press.

[6] Crowley, T. (1997). An Introduction to historical linguistics. (3rd edn). Oxford: Oxford University Press.

[7] Crystal, D. (2010). The Cambridge encyclopedia of language. (3rd ed). Cambridge: Cambridge University Press.

[8] Harper, Douglas. (2012). Online etymology dictionary. Retrieved http://www.etymonline.com (September 30, 2013).

[9] Ibn Manzoor, Abi Alfadl Almisri. (2013). Lisan al3arab. Beirut: Dar Sadir. Retrieved http://www.lisan.com (September 30, 2013).

[10] Ibn Seedah, Ali bin Ismail. (1996). AlmukhaSSaS. Beirut: Daar I2ya Alturath Al3arabi and Muassasat Altareekh al3arabi.

[11] Jassem, Zaidan Ali. (1987). Phonological variation and change in immigrant speech: A sociolinguistic study of a 1967 Arab-Israeli war immigrant speech community in Damascus, Syria. PhD Thesis, Durham University, UK. Retrieved http://etheses.dur.ac.uk/1682/1/1682.pdf (September 30, 2013).

[12] (1993). Dirasa fi 3ilmi allugha al-ijtima3i: Bahth lughawi Sauti ijtima3i fi allahajat al3arabia alshamia muqaranatan ma3a alingleeziyya wa ghairiha. Kuala Lumpur: Pustaka Antara.

[13] (1994a). Impact of the Arab-Israeli wars on language and social change in the Arab world: The case of Syrian Arabic. Kuala Lumpur: Pustaka Antara.

[14] (1994b). Lectures in English and Arabic sociolinguistics, 2 Vols. Kuala Lumpur: Pustaka Antara.

[15] (2012a). The Arabic origins of numeral words in English and European languages. International Journal of Linguistics 4 (3), 225-41. URL: http://dx.doi.org/10.5296/ijl.v4i3.1276

[16] (2012b). The Arabic origins of common religious terms in English: A lexical root theory approach. International Journal of Applied Linguistics and English Literature 1 (6), 59-71. URL: http://dx.doi.org/10.7575/ijalel.v.1n.6p.59

[17] (2012c). The Arabic origins of English pronouns: A lexical root theory approach. International Journal of Linguistics 4 (4), 83-103. URL: http://dx.doi.org/10.5296/ijl.v4i4.227.

[18] (2012d). The Arabic origins of determiners in English and European languages: A lexical root theory approach. Language in India 12 (11), 323-359. URL: http://www.languageinindia.com.

[19] (2012e). The Arabic Origins of Verb "To Be" in English, German, and French: A Lexical Root Theory Approach. International Journal of Applied Linguistics and English Literature 1 (7), 185-196. URL: http://dx.doi.org/10.7575/ijalel.v.1n.7p.185.

[20] (2012f). The Arabic origins of number and gender markers in English, German, French, and Latin: a lexical root theory approach. Language in India 12 (12), 89-119. URL: http://www.languageinindia.com.
[21] (2013a). The Arabic origins of derivational morphemes in English, German, and French: A lexical root theory approach. Language in India 13 (1), 48-72. URL: http://www.languageinindia.com.

[22] (2013b). The Arabic origins of negative particles in English, German, and French: A lexical root theory approach. Language in India 13 (1), 234-48. URL: http://www.languageinindia.com.

[23] (2013c). The English, German, and French cognates of Arabic back consonants: A lexical root theory approach. International Journal of English and Education 2 (2): 108-128. URL: http://www.ijee.org.

[24] (2013d). The Arabic origins of "water and sea" terms in English, German, and French: A lexical root theory approach. Language in India 13 (2): 126-151. URL: http://www.languageinindia.com.

[25] (2013e). The Arabic origins of "air and fire" terms in English, German, and French: A lexical root theory approach. Language in India 13 (3): 631-651. URL: http://www.languageinindia.com.

[26] (2013f). The Arabic origins of "celestial and terrestrial" terms in English, German, and French: A lexical root theory approach. International Journal of English and Education 2 (2): 323-345. URL: http://www.ijee.org.

[27] (2013g). The Arabic origins of "animal" terms in English and European languages: A lexical root theory approach. Language in India 13 (4): 68-106. URL: http://www.languageinindia.com.

[28] (2013h). The Arabic origins of "body part" terms in English and European languages: A lexical root theory approach. International Journal of Current Applied Linguistics and English Literature (1). URL: http://www.bretj.com

[29] (2013i). The Arabic origins of "speech and writing" terms in English and European languages: A lexical root theory approach. Language in India 13 (5): 108-159. URL:

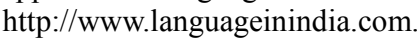

[30] (2013j). The Arabic origins of "time words" in English and European languages: A lexical root theory approach. Language in India 13 (6): 274-97. URL: http://www.languageinindia.com.

[31] (2013k). The Arabic origins of "family words" in English and European languages: A lexical root theory approach. International Journal of English and Education 2 (3): 261-77. URL: http://www.ijee.org.

[32] (20131). The Arabic origins of "personal pronouns words" in English, German, and French: A lexical root theory approach (In Arabic). 8th International Conference of Arabic Speech Renewal, Imam Bonjul University, Indonesia, 28-31 August 2013. URL: http://www.ijee.org.

[33] (2013m). The Arabic origins of "cutting and breaking words" in English and European languages: A lexical root theory approach. Research Journal of English Language and Literature 1 (2): 155-68. URL: http://www.rjelal.com.

[34] (2013n). The Arabic origins of "movement and action words" in English and European languages: A lexical root theory approach. Research Journal of English Language and Literature 1 (3): 187-202. URL: http://www.rjelal.com. 
[35] (2013o). The Arabic origins of "perceptual and sensual words" in English and European languages: A lexical root theory approach. Research Journal of English Language and Literature 1 (4): 212-24. URL: http://www.rjelal.com.

[36] (2013p). The Arabic origins of "cognitive and mental words" in English and European languages: A lexical root theory approach. International Journal of English and Education 2 (4): 62-83. URL: http://www.ijee.org.

[37] Pagel, Mark et al (2013). Ultraconserved words point to deep language ancestry across Eurasia. Proceedings of the National Academy of Sciences of the United States of America.

Retrieved www.pnas.org/content/early/2013/05/01/128726110 (July 1, 2013)
[38] Pyles, T. and J. Algeo. (1993). The origins and development of the English language. (4th edn). San Diego: HBJ.

[39] Roach, P. (2008). English phonetics and phonology: A practical course. (4th edn). Cambridge: Cambridge University Press.

[40] Ruhlen, M. (1987). A guide to the world's languages: Classification, vol 1. London: Arnold.

[41] (1994). On the origin of languages: Studies in linguistic taxonomy. Stanford, Ca.: Stanford University Press.

[42] Yule, G. (2006). The study of language. (3rd ed). Cambridge: Cambridge University Press. 